\title{
1 Ubiquilin proteins regulate EGFR levels and activity in lung
}

\section{2 adenocarcinoma cells}

3 Running title: Regulation of EGFR by UBQLN proteins

4 Authors: Zimple Kurlawala ${ }^{1 * 5}$, Kumar Saurabh ${ }^{1 *}$, Rain Dunaway ${ }^{2}$, Parag P. Shah ${ }^{1}$, Leah

5 J. Siskind ${ }^{1,3}$ and Levi J. Beverly ${ }^{1,3,4, \#}$

6 Affiliations:

7 1James Graham Brown Cancer Center, School of Medicine, University of Louisville, 8 Louisville, KY USA.

9 2School of Medicine, University of Louisville, KY, USA

10 '3epartment of Pharmacology and Toxicology, University of Louisville, Louisville, KY, 11 USA.

12 "Division of Hematology and Oncology, School of Medicine, University of Louisville, 13 Louisville, KY, USA.

145 Laboratory of Molecular Gerontology, National Institute on Aging, National Institutes of 15 Health, Baltimore, MD, USA (author's present address).

16 *Equal authors

$17{ }^{\#}$ Corresponding author (Email: levi.beverly@louisville.edu)

\section{Abstract}

20 Ubiquilin proteins (UBQLNs) are involved in diverse cellular processes like ERAD

21 (endoplasmic reticulum associated degradation), autophagy, apoptosis and epithelial to 22 mesenchymal transition. UBQLNs interact with a variety of substrates, including cell

23 surface receptors, transcription factor regulators, proteasomal machinery proteins, and

24 transmembrane proteins. Additionally, previous work from our lab shows that UBQLN1 25 interacts with IGFR family members (IGF1R, IGF2R, INSR) and this interaction regulates 26 the activity and proteostasis of IGFR family members. Here, we examined regulation of 27 UBQLN1 with Epidermal Growth Factor Receptor (EGFR) in lung adenocarcinoma cells. 
28 Loss of UBQLN1 occurs at high frequency in human lung cancer patient samples and we

29 have shown that loss of UBQLN1 is capable altering processes involved in cell

30 proliferation, migration, invasion and epithelial to mesenchymal transition in lung

31 adenocarcinoma cell lines. Here, we present data that loss of UBQLN1 resulted in

32 increased turnover of total EGFR, whilst increasing the relative amount of active EGFR

33 in lung adenocarcinoma cells, especially in the presence of its ligand EGF. Furthermore,

34 loss of UBQLN1 led to a more invasive cell phenotype as manifested by increased

35 proliferation, migration and speed of movement of these lung adenocarcinoma cells.

36 Taken together, UBQLN1 regulates expression and stability of IGFRs and EGFR,

37 members of the receptor tyrosine kinase family of proteins in lung cancer cells.

38 Key Words: UBQLN1, Ubiquilin, EGFR, cancer, IGFR

Introduction

41 Cancer and Alzheimer's disease (AD) are seemingly caused by contrasting cellular

42 processes; aberrant cell survival for cancer and aberrant cell death for AD (Shafi, 2016).

43 The family of adapter proteins, Ubiquilins (UBQLNs), are lost in multiple types of cancers

44 as well as in AD (Beverly, Lockwood, Shah, Erdjument-Bromage, \& Varmus, 2012;

45 Viswanathan et al., 2011; Y. Wang et al., 2015). This family consists of five members;

46 UBQLN1-4 and UBQLNL, which contains an N-terminus ubiquitin-like (UBL) domain and

47 a C-terminus ubiquitin-associated (UBA) domain (Kleijnen et al., 2000; Z. Kurlawala,

48 Shah, Shah, \& Beverly, 2017; Marin, 2014). Ubiquilin1 is involved in a variety of cellular

49 processes like ERAD (endoplasmic reticulum associated degradation) (Lim et al., 2009;

50 Shah et al., 2015), autophagy (Lee, Arnott, \& Brown, 2013; Elsa-Noah N'Diaye et al., 
51 2009), apoptosis (Sun et al., 2015) and epithelial to mesenchymal transition (EMT) (Shah

52 et al., 2015; Yadav et al., 2017). Ubiquilin1 also interacts with diverse substrates -

53 proteins involved in the proteasomal machinery (PSMD4, BAG6) (Z. Kurlawala, Shah, et

54 al., 2017) cell surface receptors, GABA-A (Saliba, Pangalos, \& Moss, 2008), GPCR's (E.

55 N. N'Diaye et al., 2008), PSEN1/2 (Mah, Perry, Smith, \& Monteiro, 2000; Massey et al.,

56 2004), IGF1R (Z. Kurlawala, Dunaway, et al., 2017; Z. Kurlawala, Shah, et al., 2017),

57 transcription factor regulators, IKBa (Feng et al., 2004) and other transmembrane proteins

58 ESYT2 (Z. Kurlawala, Shah, et al., 2017), CD47 (Wu, Wang, Zheleznyak, \& Brown, 1999)

59 and BCLb (Beverly et al., 2012). Ubiquilin1 is a versatile, multi-purpose adaptor that

60 interacts with a wide range of substrates; thus, it can regulate multiple important cellular

61 processes.

62 UBQLN2, is another UBQLN family member that is constitutively expressed in most cell

63 types and shares more than $75 \%$ homology with UBQLN1, indicating that it likely shares

64 similar biological functions(Marin, 2014). Like UBQLN1, UBQLN2 also has a UBL domain

65 which interacts with the proteasome and a UBA domain which recognizes ubiquitin on

66 target proteins (Kleijnen, Alarcón, \& Howley, 2003; Renaud, Picher-Martel, Codron, \&

67 Julien, 2019). Additionally, UBQLN2 has a 12-PXX repeat region which makes it unique

68 among the UBQLN family proteins (Renaud et al., 2019).

69 Receptor tyrosine kinases (RTKs) are cell surface receptors found to be responsible for

70 mediating signaling pathways crucial to cell proliferation, cell migration and invasion of

71 many types of cancer (Zwick, Bange, \& Ullrich, 2001). Our lab was first to identify

72 interaction of UBQLN1 with a RTK family member, namely insulin-like growth factor

73 receptors (IGFRs) (Z. Kurlawala, Dunaway, et al., 2017). Loss of UBQLN1 leads to a 
74 significant decrease in the amount of total IGF1R, an increase in phosphorylated IGF1R

75 and dramatic increases in their migratory potential when stimulated with IGF in lung

76 adenocarcinoma. Epidermal growth factor receptor (EGFR), a transmembrane protein

77 that is also a member of the RTK family, is mutated or over-expressed in multiple cancers

78 and AD (Lurje \& Lenz, 2009; Porta et al., 2011; Tavassoly, Sato, \& Tavassoly, 2020).

79 EGFR is one of the most commonly studied oncogenes to date. It is often upregulated in

80 multiple cancers, and downregulated in AD (Shafi, 2016). This unique inverse relationship

81 between cancer and AD has been classified for a wide array of proteins, including p53,

82 IGF1R and BCL2 (Shafi, 2016). EGFR is a well-established therapeutic target of kinase

83 inhibitors (gefitinib, erlotinib, and lapatinib) and monoclonal antibodies (cetuximab,

84 panitumumab, and trastuzumab). Many of these therapeutics are now being used as first

85 line treatment for both lung cancer and Alzheimer's patients (Lurje \& Lenz, 2009; Porta

86 et al., 2011; Tavassoly et al., 2020). In this study, we present data establishing the

87 interaction of UBQLN1 and UBQLN2 with EGFR. In lung adenocarcinoma cell lines,

88 downregulation of UBQLN1, followed by EGF stimulation, leads to degradation of total

89 EGFR protein, and an increase in migration and invasion potential of these cells.

91 Materials and Methods

92 Cell Culture, Transfection and EGF stimulation

93 Human embryonic kidney 293T (HEK293T) cells were procured from American Type

94 Culture Collection (ATCC, Rockville, MD, USA) and cultured in DMEM medium

95 (\#SH30243, Hyclone, Logan, UT, USA) supplemented with 10\% fetal bovine serum

96 (\#SH30070, Hyclone, Logan, UT, USA) and 1\% antibiotic/antimycotic (\#SV30010, 
97 Hyclone, Logan, UT, USA) at $37^{\circ} \mathrm{C}$ with $5 \% \mathrm{CO}_{2}$. A549 (lung adenocarcinoma line) were

98 procured from ATCC and cultured in RPMI (\#SH30027, Hyclone, Logan, UT, USA)

99 supplemented with $10 \%$ FBS, $1 \%$ antibiotic/antimycotic. siRNA transfections were

100 performed as described previously(Z. Kurlawala, Dunaway, et al., 2017). ${ }^{19}$ Briefly, 48

101 hours after transfection, cells were serum starved (SS) for 3 hours, incubated with protein

102 synthesis inhibitor, Cycloheximide $(\mathrm{CH}, 10 \mu \mathrm{M})$ for 1 hour prior to supplementing serum-

103 free media with 25 or $50 \mathrm{ng} / \mathrm{ml}$ based on the experiment. EGF (\#PHG0314, Thermo Fisher

104 Scientific, Waltham, MA, USA). 3 hours later, cells were harvested and probed for EGFR

105 (total and phosphorylated) protein by Western Blot analysis. For dose and time-

106 dependent studies, cells were stimulated and/or incubated for doses and time-points

107 indicated in respective figures.

108 Plasmid Construction

109 As described previously, constructs with deleted domains of UBQLN1 (Figure 1A), were

110 developed using Q5 Site-Directed mutagenesis kit as per manufacturers protocol (New

111 England Biolabs \# E0554; Ipswich, MA, USA) and confirmed by sequencing(Z. Kurlawala,

112 Shah, et al., 2017). ${ }^{7}$

113 Immunoprecipitation, Protein estimation and Western Blot

114 Immunoprecipitation (IP) was performed as described previously. Harvested cells for 115 each procedure (IP and/or transfection) were lysed with 1\% CHAPS lysis buffer and total 116 protein was estimated using the BCA quantification method(Z. Kurlawala, Shah, et al., 117 2017). Western blot analyses were performed in Bolt Bis-Tris gels (\#BG4120BOX, Life 118 Technologies, Grand island, NY, USA) as per manufacturer's protocol using antibodies 119 from Santa Cruz, Dallas, TX, USA (GAPDH \# sc47724); Sigma-Aldrich, St. Louis, MO, 
USA (Actin \# A5316); Yenzym Antibodies LLC, South San Francisco, CA, USA (UBQLN

121 polyclonal was made by inoculating rabbits with a peptide specific to UBQLN1); and Cell

122 Signaling, Danvers, MA, USA (UBQLN1 \# 14526, FLAG \# 14793, EGFR \# 4267, pEGFR

123 Tyr1068 \# 3777).

124 Cell viability and Migration assay

125 Cell viability and migration assays were performed as described earlier. ${ }^{19}$ Briefly, A549 126 cells were cultured in $60 \mathrm{~mm}$ culture plates. After 12 hours of transfection with siRNA, cells

127 were trypsinized, counted and 2000 cells were reseeded per well in 96-well plates in 128 complete media. 12 hours post-reseeding in complete media, cells were serum starved

129 for 3 hours followed by stimulation with EGF and were cultured in media containing $2 \%$ 130 FBS. Cell viability was analyzed for four successive days using AlamarBlue AM $^{\mathrm{TM}}$ 131 (\#DAL1100, Thermo Fisher Scientific, Waltham, MA, USA). At the same time following 132 transfection, 5000 cells were seeded in Transwell ${ }^{\mathrm{TM}}$ cell culture inserts (\#CLS3464, 133 Corning Inc., Corning, NY, USA) in triplicate for each condition as described previously. ${ }^{19}$

134 Briefly, cells were allowed to grow on Transwell ${ }^{\mathrm{TM}}$ cell culture insert in serum free media, 135 serum free media supplemented with EGF $(50 \mathrm{ng} / \mathrm{ml})$ and serum free media 136 supplemented with both EGF and Erlotinib (1 $\mu \mathrm{M})$. After 24hrs, membranes were washed 137 once with PBS, fixed with ethanol, stained with Giemsa stain (\#R03055, Sigma-Aldrich, 138 St. Louis, MO, USA) and cells were counted on microscope.

139 Live Cell Imaging

140 A549 cells were transfected with siRNA against UBQLN1 and non-targeting control. 24

141 hours post-transfection, cells were trypsinized, counted and 10,000 cells were reseeded 142 per well in 12-well plate, coated with thin layer commercial extracellular matrix (ECM \# 
143 E6909, Sigma-Aldrich, St. Louis, MO, USA) at a concentration of $1 \mu \mathrm{g} / \mathrm{cm}^{2}$ for 12 hours

144 (overnight) in complete media. Next day, all wells were serum starved for 3 hours. Cells

145 were then maintained in media with $2 \%$ FBS or $2 \%$ media supplemented with EGF

$146(25 \mathrm{ng} / \mathrm{ml})$. Cells were imaged for $48 \mathrm{hrs}$ on a time-interval of $15 \mathrm{mins}$ on Keyence BZ-X810.

147 All pictures were stitched together to produce a video with speed of $14 \mathrm{fps}$. For dynamic

148 tracking, 5 single cells were analyzed on Keyence BZ-X810 software to generate

149 Chemotaxis plot and to calculate movement and speed of cells.

150 RNAi Sequences

151 All RNAi (siRNAs) used for study were ordered from Thermo Fisher Scientific Biosciences

152 Inc. Lafayette, CO 80026, USA and transfections were done using Dharmafect1 as per

153 the supplier's instructions.

154 1. Non-Targeting Control

155 UAAGGCUAUGAAGAGAUACAA

156 2. UBQLN1

157 siU11: GAAGAAAUCUCUAAACGUUUUUU

158 siU12: GUACUACUGCGCCAAAUUUUU

159 Statistical Analysis

160 All statistics were performed using GraphPad Prism 8 software. Unless otherwise 161 specified, significance was determined by one-way ANOVA, using a cut off of $p<0.05$.

163 Results

164 Ubiquilin1 and Ubiquilin2 interact with EGFR 
165 Our previous data described that UBQLN1 interacts with IGF1R. In this study, we aimed

166 to explore the possibility that UBQLN proteins might interact with and regulate additional

167 RTK family members, like EGFR. We first performed co-immunoprecipitation (IP) of 168 UBQLNs to identify interacting proteins (Figure 1). We transiently transfected HEK293T

169 cells with UBQLN1-FLAG or UBQLN2-FLAG constructs, then pulled down UBQLN-

170 interacting proteins using anti-FLAG conjugated agarose beads. Western blot analysis of

171 these FLAG-IP samples revealed that UBQLN1 and UBQLN2 interacted with total EGFR

172 (Figure 1B). Like UBQLN1, UBQLN2 has an N-terminus UBL, a C-terminus UBA and four

173 STI (STI1-4) domains, plus UBQLN2 has an additional, unique PXX (12 tandem repeats)

174 domain (Figure 1A). To determine the interacting domain of UBQLN1, we created two

175 constructs as described in Figure $1 \mathrm{~A}$ and performed similar co-immunoprecipitation

176 experiment. For one construct, we deleted the UBA domain of UBQLN1 (labeled 542X),

177 and for the second construct we deleted the UBL domain (labeled 112X). Our results

178 indicated that the UBL domain is the primary site of interaction with EGFR. Deletion of

179 the UBA domain did not cause a loss of interaction between UBQLN1 and EGFR (Figure 180 1C).

181 Ubiquilin1 regulates expression and activity of EGFR

182 Next, we investigated whether loss of UBQLN1 regulated EGFR expression and activity

183 in lung adenocarcinoma cells (Figure 2). A549 cells were transiently transfected with non-

184 targeting siRNA (NT) and two UBQLN1-specific siRNAs (Figure 2A). Cells were then

185 serum starved (SS) to synchronize the cell cycle and remove all confounding growth

186 factors present in serum. Then cells were treated with cycloheximide $(\mathrm{CH})$ to prevent 187 synthesis of new EGFR protein. Upon loss of UBQLN1, there was a decrease in total 
188 EGFR expression compared to the NT control. This decrease in total EGFR expression was significantly enhanced when the receptor was stimulated with EGF ligand. Next, we

190 tested regulation of total EGFR when stimulated with different doses of EGF ligand

191 (Figure 2B). There was significantly increased degradation of total EGFR in cells lacking

192 UBQLN1 compared to controls at $10 \mathrm{ng} / \mathrm{ml}$, which was enhanced at $100 \mathrm{ng} / \mathrm{ml}$.

193 Interestingly, expression of phosphorylated EGFR was not as sensitive to the loss of 194 UBQLN1. Therefore, in cells lacking UBQLN1, there was an EGF dose-dependent 195 degradation of EGFR.

196 Next, we performed experiments to determine temporal regulation of EGFR by UBQLN1

197 (Figure 2D, E). Lung adenocarcinoma cells were transfected with non-targeting or 198 UBQLN1-specific siRNAs. Two days post-transfection, cells were serum starved for 3 199 hours, then stimulated with EGF $(50 \mathrm{ng} / \mathrm{ml})$ for indicated time points. Upon stimulation with 200 EGF, we observed degradation of total EGFR as time progressed. However, in cells 201 lacking UBQLN1, there was significantly increased degradation compared to control as 202 time progressed.

203 Ubiquilin1-deficient cells exhibit increased cell viability and migration potential

204 EGFR proteins play a role in maintaining cell viability and stimulating migratory potential 205 of lung adenocarcinoma cells. We investigated the influence of loss of UBQLN1 on these 206 EGFR mediated processes (Figure 3). A549 cells were transiently transfected with siRNA 207 for Ubiquilin1 and non-targeting control and cultured in different conditions as indicated 208 (complete media, serum starvation (SS) for 3 hours, SS + EGF (50ng/ml), 209 SS+EGF+Erlotinib, a phospho-EGFR inhibitor, 1uM). Cells were harvested after 3 hours 210 and analyzed by Western Blot. When stimulated with EGF, Ubiquilin1 deficient cells 
211 showed almost complete loss of total EGFR and increased expression of phosphorylated

212 EGFR, which was blocked by Erlotinib (Figure 3A). Next, A549 cells were transfected with

213 non-targeting or UBQLN1-specific siRNAs, and cell viability was measured for four

214 consecutive days using alamarBlue ${ }^{\mathrm{TM}}$ (Figure 3B). Consistent with our previous findings,

215 loss of UBQLN1 resulted in increased cell growth in lung adenocarcinoma cell lines.

216 Interestingly, there was an increase in the relative number of cells in UBQLN1 deficient

217 cells stimulated with EGF, compared to controls. Next, we determined the effects of loss

218 of UBQLN1 on cell migration (Figure 3C, D). Using a Transwell ${ }^{\mathrm{TM}}$ migration plate, we

219 seeded A549 cells that had been transfected with either non-targeting or UBQLN1-

220 specific siRNAs and cultured the cells under three conditions - serum-free media (SF);

221 SF media supplemented with EGF; and SF media supplemented with EGF and Erlotinib,

222 a phospho-EGFR inhibitor. Consistent with our previous data using this migration model,

223 UBQLN1 deficient cells exhibited an approximately 3-fold increase in migration when

224 stimulated with EGF. Erlotinib decreased migration of cells in both control and UBQLN1

225 deficient cells.

226 Loss of Ubiquilin1 results in increased cell movement and speed

227 We examined individual cell movement and speed to explore increased migratory

228 potential in cells lacking UBQLN1 (Figure 4). We utilized live cell imaging equipped with

229 image analysis software (Keyence). A549 lung adenocarcinoma cells were transfected

230 with non-targeting or UBQLN1-specific siRNAs for 24 hours after which they were serum

231 starved for another 24 hours. At this point, 10,000 cells per well (12-well plate) were

232 seeded on a thin layer of commercial extracellular matrix (ECM) in the presence or

233 absence of EGF (25ng/ml) to yield the following conditions: siNT (+/-) EGF and siUBQLN1 
234 (+/-) EGF. Live cell images were captured for 48 hours using a Keyence microscope. All 235 captured images were stitched together to make a representative video (Figure S1). As 236 evident from the videos, loss of UBQLN1 resulted in increased movement of A549 cells 237 which was further enhanced by EGF stimulation. These data are represented as

238 chemotaxis plots (Figure 4A). Additionally, we quantified the distance traveled and the 239 rate of travel (speed) for each cell (Figure 4B). These data show that cells lacking 240 UBQLN1 travel further and move faster as compared to the non-targeting controls. These 241 differences were enhanced in the presence of EGF.

\section{Discussion}

244 UBQLN1 was reported to be lost in approximately fifty percent of non-small cell lung 245 adenocarcinomas. Our group is interested in understanding how loss of function of 246 UBQLN proteins contributes to the metastatic progression of human lung 247 adenocarcinoma.(Z. Kurlawala, Dunaway, et al., 2017; Shah et al., 2015; Yadav et al., 248 2017). Previously we have shown that interaction between UBQLN1 with IGF1R results 249 in stabilization of this receptor. When UBQLN1 is lost, it leads to increased 250 phosphorylation of the auto-phosphorylation site on the IGF receptor, while total IGF1R 251 levels decreased. Similarly, in this manuscript, we report that loss of UBQLN1 does not 252 alter phosphorylated EGFR expression while causing a robust decrease in total EGFR 253 expression. Additionally, we have reported dose and time dependent decreases in total 254 IGF1R when stimulated with IGF ligand (Z. Kurlawala, Dunaway, et al., 2017). Likewise, 255 in this study, lung adenocarcinoma cells lacking UBQLN1 also exhibit an enhanced 256 degradation of EGFR when stimulated with EGF that is both dose and time dependent. 
257 These data suggest that loss of UBQLN1 accelerates both EGFR and IGF1R turnover in

258 cancer cells. UBQLN1 might be crucial to maintain the stability of these RTKs ( $Z$

259 Kurlawala \& Beverly, 2017), thus influencing pro-growth and pro-survival signaling

260 pathways in lung adenocarcinoma cells.

261 We have previously demonstrated that downregulation of UBQLN1 leads to significantly

262 increased expression of mesenchymal markers like Vimentin, Snail and ZEB1 indicating

263 that UBQLN1 may play a role in suppression of metastasis in lung cancer (Shah et al.,

264 2015). Additionally, knockdown of UBQLN1 by siRNA or mir155-mediated

265 downregulation of UBQLN1 in lung cancer cells promoted an EMT-like phenotype. Data

266 presented here further supports the role of UBQLN1 in proliferation and migration of

267 cancer cells and this was exacerbated in the presence of EGF stimulation. These results,

268 when considered in conjunction with our previous work with IGF1R, suggest that cells

269 lacking UBQLN1, then stimulated with IGF or EGF, enhanced the metastatic potential of

270 cancer cells(Z. Kurlawala, Dunaway, et al., 2017). Our results point to a destabilization of

271 these RTKs when UBQLN1 is lost which further leads to an invasive phenotype in lung

272 adenocarcinoma cells. In a related study, UBQLN4, another member of the UBQLN

273 family, was shown to interact with RNF11, an E3 ubiquitin ligase of p21. Overexpression

274 of UBQLN4 induced cellular senescence and cell cycle arrest in gastric cancer cells (S.

275 Huang et al., 2019). These findings further support significance of UBQLN proteins in

276 cancer progression and tumorigenesis. As reported here, cells that lack UBQLN1 show

277 increased cell movement and speed captured with live cell imaging over multiple days.

278 The overall movement and speed of these lung adenocarcinoma cells was further

279 accelerated in the presence of EGF stimulation. RTK family of kinases play critical roles 
280 in progression of human lung cancer. In non-small cell lung cancer (NSCLC), IGFR and

281 EGFR are overexpressed and UBQLNs are underexpressed and synergistically

282 contribute to tumor development and progression (Guo et al., 2017; Oliveira, Schiffelers,

283 Storm, Henegouwen, \& Roovers, 2009). Collectively, these data indicate a critical role for

284 UBQLNs in the normal proteolytic degradation of RTKs and loss of UBQLN function in

285 cancer cells leads to aberrant RTK-mediated signaling, further enhancing the metastatic

286 potential of these cancers. Downstream pathways activated by these cell surface

287 receptors crosstalk and upon mutual activation lead to acquired resistance against EGFR-

288 targeted drugs. We propose that targeting both EGF and IGF receptors at once might

289 enhance anti-tumor efficacy and would be a promising approach for NSCLC

290 therapies(Guo et al., 2017; Oliveira et al., 2009; Yeo et al., 2015). Being able to target

291 both of these pathways simultaneously via their interaction with UBQLNs is an exciting

292 avenue to explore.

293 Our lab is also interested in the phenomenon of inverse relation of cancer with

294 neurodegenerative disorders. Single nucleotide polymorphisms (SNPs) in UBQLN1 gene

295 are associated with late onset of Alzheimer's Disease (AD) (Bertram et al., 2005).

296 Additionally, mutations found in UBQLN2, provide a possible pathophysiological link for

297 worse prognosis in amyotrophic lateral sclerosis/frontotemporal dementia

298 (ALS/FTD)(Brettschneider et al., 2012; Renaud et al., 2019). After UBQLN1 was found to

299 interact with the presenilin complex via a yeast-two hybrid screen, research efforts were

300 quickly underway to establish the role that UBQLN family members might play in

301 neurodegenerative diseases(Bertram et al., 2005; Brettschneider et al., 2012; Hiltunen et

302 al., 2006; Mah et al., 2000; Renaud et al., 2019; Viswanathan et al., 2011). Perhaps, 
UBQLN family members inversely regulate outcomes in cancer and neurodegenerative diseases such that loss of function of UBQLN proteins in epithelial cells leads to a cancerous phenotype while in post-mitotic neurons leads to a degenerative phenotype. UBQLNs could play a regulatory role in presenilin complex assembly by directly, or indirectly, altering the stability of individual proteins found in these complexes thereby destabilizing the complex as a whole and leading to the pathogenesis of the disease state. Further experiments would be needed in order to establish the exact role.

310 Interestingly, some members of the BCL2 family, IGF1R, and EGFR are overexpressed 311 in cancer but under-expressed in brains of AD patients (Shafi, 2016). Interestingly, each 312 of these proteins, either in this study or in our previous work, have been shown to be 313 stabilized by UBQLN1 (Beverly et al., 2012; Z Kurlawala \& Beverly, 2017). In AD, $\beta$ 314 amyloid aggregations accumulate in the brain. Studies have shown that inhibiting IGF1R 315 and EGFR leads to significant reduction in $\beta$-amyloid aggregations (Wang et al. 2012; 316 Gontier et al., 2015). Polyubiquitination of EGFR regulates its cellular location and 317 stability, and this ubiquitination can be decreased by mutating the lysine residues without 318 having an effect on the tyrosine kinase activity of EGFR(F. Huang, Kirkpatrick, Jiang, 319 Gygi, \& Sorkin, 2006). Thus, inhibition of RTKs could not only benefit certain subsets of 320 cancer, it could also be beneficial in several neurodegenerative diseases(Gontier, 321 George, Chaker, Holzenberger, \& Aïd, 2015; Tavassoly et al., 2020; L. Wang et al., 2012).

322 These studies further support our belief that UBQLNs might be regulating multiple 323 diseases indirectly.

324 In conclusion, the UBA-UBL domain-containing family of UBQLNs facilitate normal 325 proteasomal degradation, and substrate selection by UBQLNs is critical in cancer and 
326 neurodegenerative diseases. Our IP data provides evidence for interaction of both

327 UBQLN1 and UBQLN2 (shares more than 75\% homology with UBQLN1) with EGFR.

328 Taken together with our previous findings, both IGFRs and EGFR were detected as

329 interacting partners of UBQLN1. The lung adenocarcinoma cells lacking UBQLN1 exhibit

330 enhanced degradation of both EGFR and IGF1R, especially in the presence of their

331 respective ligands. Thus, UBQLN1 is important in maintaining stability of RTKs, like

332 EGFR and IGF1R. Loss of UBQLN1 alters the normal degradative fate of these receptors

333 leading to downstream alterations in signaling that result in a cellular phenotype with 334 enhanced proliferation, migration and movement of the cells. The RTK family of proteins

335 are highly regulated in both cancer and neurodegenerative diseases and this sets the 336 stage for the development of directed therapies. Our work with UBQLNs indicates that

337 they are uniquely poised as therapeutic targets given their role in regulating the stability 338 of multiple members of the RTK family, and this regulation might be inversely impacted 339 in cancer versus neurodegenerative diseases.

\section{List of Abbreviations}

342 AD: Alzheimer's disease; UBQLNs: Ubiquilin family of adapter proteins; UBA: Ubiquitin-

343 associated domain; UBL: Ubiquitin-like domain; APP: $\beta$-amyloid precursor protein; ER:

344 Endoplasmic reticulum; EMT: Epithelial to mesenchymal transition; BCL2: B-cell

345 lymphoma 2; RTK: Receptor tyrosine kinase; IGFR: Insulin-like growth factor receptor;

346 EGFR: Epidermal growth factor receptor; ALS: Amyotrophic lateral sclerosis; NSCLC:

347 Non-small cell lung cancer; EGF: Epidermal growth factor (ligand). 


\section{Declarations}

350 Consent for publication

351 Not applicable

352 Availability of data and materials

353 The datasets used and/or analyzed during the current study are available from the

354 corresponding author upon reasonable request.

355 Competing interests

356 The authors declare that they have no competing interests

\section{Funding}

358 This work was supported by NIH R01CA193220, Kentucky Lung Cancer Research

359 Program (KLCRP) and funds from James Graham Brown Cancer Center, University of

360 Louisville to LJB. RD was supported by NIH National Cancer Institute Cancer Education

361 Program grant R25-CA134283. The funding bodies have no role in the design of the

362 study; collection, analysis, and interpretation of data; and in writing the manuscript.

\section{Authors' contributions}

364 KS, ZK, RD and PPS did the experiments. KS, ZK, LJS and LJB conceived studies, did

365 the analysis and wrote the manuscript. All authors have read and approved the 366 manuscript.

367 Acknowledgements

368 We are grateful to the Beverly and Siskind lab members, especially Lavona Casson and 369 Mark Doll, for continuous support and sharing of resources and insight.

\section{References}


372 Bertram, L., Hiltunen, M., Parkinson, M., Ingelsson, M., Lange, C., Ramasamy, K., . . . Tanzi, R. E. (2005). Family-based association between Alzheimer's disease and variants in UBQLN1. N Engl J Med, 352(9), 884-894. doi:10.1056/NEJMoa042765

375

376

377

378

379

380

381

382

383

384

385

386

387

388

389

390

391

392

393

394

Beverly, L. J., Lockwood, W. W., Shah, P. P., Erdjument-Bromage, H., \& Varmus, H. (2012). Ubiquitination, localization, and stability of an anti-apoptotic BCL2-like protein, BCL2L10/BCLb, are regulated by Ubiquilin1. Proc Natl Acad Sci U S A, 109(3), E119-126. doi:10.1073/pnas.1119167109

Brettschneider, J., Van Deerlin, V. M., Robinson, J. L., Kwong, L., Lee, E. B., Ali, Y. O., . . . Elman, L. (2012). Pattern of ubiquilin pathology in ALS and FTLD indicates presence of C9ORF72 hexanucleotide expansion. Acta Neuropathol, 123(6), 825839.

Feng, P., Scott, C. W., Cho, N. H., Nakamura, H., Chung, Y. H., Monteiro, M. J., \& Jung, J. U. (2004). Kaposi's sarcoma-associated herpesvirus K7 protein targets a ubiquitin-like/ubiquitin-associated domain-containing protein to promote protein degradation. Mol Cell Biol, 24(9), 3938-3948. Retrieved from https://www.ncbi.nlm.nih.gov/pubmed/15082787

Gontier, G., George, C., Chaker, Z., Holzenberger, M., \& Aïd, S. (2015). Blocking IGF signaling in adult neurons alleviates Alzheimer's disease pathology through amyloid- $\beta$ clearance. Journal of Neuroscience, 35(33), 11500-11513.

Guo, X.-F., Zhu, X.-F., Cao, H.-Y., Zhong, G.-S., Li, L., Deng, B.-G., . . Zhen, Y.-S. (2017). A bispecific enediyne-energized fusion protein targeting both epidermal growth factor receptor and insulin-like growth factor 1 receptor showing enhanced antitumor efficacy against non-small cell lung cancer. Oncotarget, 8(16), 27286. 
Hiltunen, M., Lu, A., Thomas, A. V., Romano, D. M., Kim, M., Jones, P. B., . . . Berezovska, O. (2006). Ubiquilin 1 modulates amyloid precursor protein trafficking and $A \beta$ secretion. Journal of Biological Chemistry, 281(43), 32240-32253.

Huang, F., Kirkpatrick, D., Jiang, X., Gygi, S., \& Sorkin, A. (2006). Differential regulation of EGF receptor internalization and degradation by multiubiquitination within the kinase domain. Molecular Cell, 21(6), 737-748.

Huang, S., Li, Y., Yuan, X., Zhao, M., Wang, J., Li, Y., . . . Huang, C. (2019). The UbLUBA Ubiquilin4 protein functions as a tumor suppressor in gastric cancer by p53dependent and p53-independent regulation of p21. Cell Death Differ, 26(3), 516530. doi:10.1038/s41418-018-0141-4

Kleijnen, M. F., Alarcón, R. M., \& Howley, P. M. (2003). The ubiquitin-associated domain of hPLIC-2 interacts with the proteasome. Molecular biology of the cell, 14(9), 3868-3875.

Kleijnen, M. F., Shih, A. H., Zhou, P., Kumar, S., Soccio, R. E., Kedersha, N. L., . . . Howley, P. M. (2000). The hPLIC proteins may provide a link between the ubiquitination machinery and the proteasome. Molecular Cell, 6(2), 409-419.

Kurlawala, Z., \& Beverly, L. (2017). Ubiquilin Proteins are critical adaptors that regulate proteostasis. Journal of Cell Signaling, 2, 145.

Kurlawala, Z., Dunaway, R., Shah, P. P., Gosney, J. A., Siskind, L. J., Ceresa, B. P., \& Beverly, L. J. (2017). Regulation of insulin-like growth factor receptors by Ubiquilin1. Biochem J, 474(24), 4105-4118. doi:10.1042/BCJ20170620 
416 Kurlawala, Z., Shah, P. P., Shah, C., \& Beverly, L. J. (2017). The STI and UBA Domains of UBQLN1 are Critical Determinants of Substrate Interaction and Proteostasis. J Cell Biochem. doi:10.1002/jcb.25880

Lee, D. Y., Arnott, D., \& Brown, E. J. (2013). Ubiquilin4 is an adaptor protein that recruits Ubiquilin1 to the autophagy machinery. EMBO Rep, 14(4), 373-381. doi:10.1038/embor.2013.22

Lim, P. J., Danner, R., Liang, J., Doong, H., Harman, C., Srinivasan, D., . . Monteiro, M.

Mah, A. L., Perry, G., Smith, M. A., \& Monteiro, M. J. (2000). Identification of ubiquilin, a novel presenilin interactor that increases presenilin protein accumulation. $J$ Cell Biol, 151(4),

847-862.

Retrieved

from

431

432

\section{https://www.ncbi.nlm.nih.gov/pubmed/11076969}

Marin, I. (2014). The ubiquilin gene family: evolutionary patterns and functional insights. BMC Evol Biol, 14, 63. doi:10.1186/1471-2148-14-63

Massey, L. K., Mah, A. L., Ford, D. L., Miller, J., Liang, J., Doong, H., \& Monteiro, M. J. (2004). Overexpression of ubiquilin decreases ubiquitination and degradation of presenilin proteins. J Alzheimers Dis, 6(1), 79-92. Retrieved from http://www.ncbi.nlm.nih.gov/pubmed/15004330

N'Diaye, E. N., Hanyaloglu, A. C., Kajihara, K. K., Puthenveedu, M. A., Wu, P., von Zastrow, M., \& Brown, E. J. (2008). The ubiquitin-like protein PLIC-2 is a negative 
regulator of G protein-coupled receptor endocytosis. Mol Biol Cell, 19(3), 12521260. doi:10.1091/mbc.E07-08-0775

441 N'Diaye, E. N., Kajihara, K. K., Hsieh, I., Morisaki, H., Debnath, J., \& Brown, E. J. (2009). PLIC proteins or ubiquilins regulate autophagy-dependent cell survival during nutrient starvation. EMBO reports, 10(2), 173-179.

444 Oliveira, S., Schiffelers, R., Storm, G., Henegouwen, P., \& Roovers, R. (2009). Crosstalk between epidermal growth factor receptor-and insulin-like growth factor-1 receptor signaling: implications for cancer therapy. Current cancer drug targets, 9(6), 748-

Porta, R., Sanchez-Torres, J., Paz-Ares, L., Massuti, B., Reguart, N., Mayo, C., . . . Salinas, P. (2011). Brain metastases from lung cancer responding to erlotinib: the importance of EGFR mutation. European Respiratory Journal, 37(3), 624-631.

Renaud, L., Picher-Martel, V., Codron, P., \& Julien, J. P. (2019). Key role of UBQLN2 in pathogenesis of amyotrophic lateral sclerosis and frontotemporal dementia. Acta Neuropathol Commun, 7(1), 103. doi:10.1186/s40478-019-0758-7

454 Saliba, R. S., Pangalos, M., \& Moss, S. J. (2008). The ubiquitin-like protein Plic-1 enhances the membrane insertion of GABAA receptors by increasing their stability within the endoplasmic reticulum. J Biol Chem, 283(27), 18538-18544.

458 Shafi, O. (2016). Inverse relationship between Alzheimer's disease and cancer, and other factors contributing to Alzheimer's disease: a systematic review. BMC neurology, 16(1), 236. 
461 Shah, P. P., Lockwood, W. W., Saurabh, K., Kurlawala, Z., Shannon, S. P., Waigel, S., .

462

463

464

465

466

467

468

469

470

471

472

473

474

475

476

477

478

479

480

481

482

483

. . Beverly, L. J. (2015). Ubiquilin1 represses migration and epithelial-tomesenchymal transition of human non-small cell lung cancer cells. Oncogene, 34(13), 1709-1717. doi:10.1038/onc.2014.97

Sun, Q., Liu, T., Yuan, Y., Guo, Z., Xie, G., Du, S., . . Chen, L. (2015). MiR-200c inhibits autophagy and enhances radiosensitivity in breast cancer cells by targeting UBQLN1. Int J Cancer, 136(5), 1003-1012. doi:10.1002/ijc.29065

Tavassoly, O., Sato, T., \& Tavassoly, I. (2020). Inhibition of Brain EGFR Activation: A Novel Target in Neurodegenerative Diseases and Brain Injuries. Molecular Pharmacology.

Viswanathan, J., Haapasalo, A., Bottcher, C., Miettinen, R., Kurkinen, K. M., Lu, A., . . . Hiltunen, M. (2011). Alzheimer's disease-associated ubiquilin-1 regulates presenilin-1 accumulation and aggresome formation. Traffic, 12(3), 330-348. doi:10.1111/j.1600-0854.2010.01149.x

Wang, L., Chiang, H.-C., Wu, W., Liang, B., Xie, Z., Yao, X., . . . Zhong, Y. (2012). Epidermal growth factor receptor is a preferred target for treating Amyloid- $\beta-$ induced memory loss. Proceedings of the National Academy of Sciences, 109(41), 16743-16748.

Wang, Y., Lu, J., Zhao, X., Feng, Y., Lv, S., Mu, Y., . . . Li, Y. (2015). Prognostic significance of Ubiquilin1 expression in invasive breast cancer. Cancer Biomark, 15(5), 635-643. doi:10.3233/CBM-150503

Wu, A. L., Wang, J., Zheleznyak, A., \& Brown, E. J. (1999). Ubiquitin-related proteins regulate interaction of vimentin intermediate filaments with the plasma membrane. 


\section{$\mathrm{Mol}$}

Cell,

4(4),

619-625.

Retrieved

from http://www.ncbi.nlm.nih.gov/pubmed/10549293

486

487

488

489

490

491

492

493

494

495

496

497

498

499

500

501

502

503

504

505

506

Yadav, S., Singh, N., Shah, P. P., Rowbotham, D. A., Malik, D., Srivastav, A., . . Beverly, L. J. (2017). MIR155 Regulation of Ubiquilin1 and Ubiquilin2: Implications in Cellular Protection and Tumorigenesis. Neoplasia, 19(4), 321-332. doi:10.1016/j.neo.2017.02.001

Yeo, C. D., Park, K. H., Park, C. K., Lee, S. H., Kim, S. J., Yoon, H. K., . . Kim, T.-J. (2015). Expression of insulin-like growth factor 1 receptor (IGF-1R) predicts poor responses to epidermal growth factor receptor (EGFR) tyrosine kinase inhibitors in non-small cell lung cancer patients harboring activating EGFR mutations. Lung cancer, 87(3), 311-317.

Zwick, E., Bange, J., \& Ullrich, A. (2001). Receptor tyrosine kinase signalling as a target for cancer intervention strategies. Endocrine-related cancer, 8(3), 161-173.

Figure Legends

Figure 1: Ubiquilin1 and Ubiquilin2 interact with EGFR. (A) Schematic of Ubiquilin ${ }^{\mathrm{WT}}$, Ubiqulin $1^{542 X}$, Ubiquilin $1^{112 X}$ and Ubiquilin2 ${ }^{\mathrm{WT}}$ constructs. Ubiquilin1 (590 amino acids) and Ubiquilin2 (620 amino acids) proteins have an N-terminal UBL domain, four STI chaperone-like domains in the middle and a C-terminal UBA domain. Ubiquilin2 has an additional 12-PXX repeat region. (B) HEK293T cells were transiently transfected with FLAG-tagged Ubiquilin1 (U1) and Ubiquilin2 (U2) followed by coimmunoprecipitation (IP) by anti-FLAG antibody and Western Blot analysis for total 
EGFR. Both Ubiquilin1 and Ubiquilin2 interact with T-EGFR. (C) HEK293T cells were transiently transfected with FLAG-U1WT, FLAG-U1542X, FLAG-U1112X or FLAGU2 followed by co-immunoprecipitation by anti-FLAG antibody and probed for EGFR. All 3 constructs of $\mathrm{U} 1$ interact with T-EGFR, indicating that the UBA domain is dispensable for interaction between these two proteins.

Figure 2: Ubiquilin1 regulates expression and activity of EGFR. (A) A549 cells were transiently transfected with two different siRNA's for Ubiquilin1 (siU1 ${ }^{1}$ and $\operatorname{siU} 1^{2}$ ) along with non-targeting control (siNT). Cells were serum starved (SS) for 3 hours, incubated with a protein synthesis inhibitor, Cycloheximide for 1 hour, supplemented with EGF (50ng/ml) for 3 hours and analyzed by Western Blot. When stimulated with EGF, cells with loss of Ubiquilin1 demonstrated loss of total EGFR compared to controls. (B) A549 cells were transiently transfected with siRNA for Ubiquilin1, SS for 3 hours and stimulated with different does of EGF (10 and 100ng/ml) for 1 hour. Cells lacking Ubiquilin1 showed a dose-dependent loss of total EGFR, quantified in (C) n=3, Twoway ANOVA, p<0.05. (D) A549 cells were transiently transfected with siRNA for Ubiquilin1, SS for 3 hours and stimulated with EGF $(50 \mathrm{ng} / \mathrm{ml})$ for indicated time points (0-240 minutes). As time passed, cells lacking Ubiquilin1 showed significantly increased loss of total EGFR compared to controls, quantified in (E) $n=3$, Two-way ANOVA, $p<0.05$.

Figure 3: UBQLN1 deficient cells show increased cell viability and migration potential. 
control, and cultured in different conditions as indicated (complete media, serum starvation (SS) for 3 hours, SS + EGF (50ng/ml), SS+EGF+Erlotinib 1uM). Cells were harvested after 3 hours and analyzed by Western Blot. When stimulated with EGF, Ubiquilin1 deficient cells showed almost complete loss of total EGFR and increased phosphorylated EGFR. (B) A549 cells were transiently transfected with siRNA for

$535 \quad$ Ubiquilin1 and non-targeting control. 12hrs post-transfection, cells reseeded in a 96536 well plate for $12 \mathrm{hrs}$ (overnight). Cells were then serum starved for 3 hours followed by 537 stimulation with EGF in $2 \%$ FBS and were cultured for 4 days. Alamar Blue readings were recorded every 24 hours and relative cell viability of UBQLN1 deficient cells were compared to control cells on each day. UBQLN1 deficient cells supplemented with EGF showed significantly increased viability compared to controls. One-way ANOVA, ${ }^{* *} p<0.01,{ }^{* * * *} p<0 .-0001$ (C) A549 cells were transiently transfected with siRNA for

542 Ubiquilin1, seeded in a transwell setup to assess cell migration in response to EGF 543 stimulation. Cells were cultured in the top chamber in one of 3 conditions - serum544 free media, serum-free media supplemented with EGF and serum-free media supplemented with EGF and Erlotinib. Media supplemented with 10\% FBS was used

546 as chemo-attractant in the bottom chamber. At the end of 24 hours, cells were fixed 547 and probed with HEMA 3 stain and data are quantified in (D). Under all 3 conditions, 548 UBQLN1 deficient cells demonstrated increased invasive behavior compared to 549 controls. $n=2$, Two-way ANOVA, $p<0.05$.

550

Figure 4: Loss of UBQLN1 results in increased cell movement and speed. (A) A549 
$55324 \mathrm{hrs}$ post-transfection, cells were reseeded in a plate coated with ECM $\left(1 \mu \mathrm{g} / \mathrm{cm}^{2}\right)$ for

$55412 \mathrm{hrs}$ (overnight) in complete media. Cells were then serum starved for 3 hours

555 followed by stimulation with EGF in $2 \%$ FBS. Cells were then imaged for $48 \mathrm{hrs}$ on a

556 time-interval of $15 \mathrm{mins}$. All pictures were stitched together to produce a video with

557 speed of $14 \mathrm{fps}$ (Supplementary figure 1). (B) 5 single cells were analyzed on Keyence

558 BZ-X810 to generate Chemotaxis plot. (C) Dynamic tracking were also used to 559 calculate speed and movement of 5 single cells. One-way ANOVA, ${ }^{*} p<0.05$, $560 \quad{ }^{* * * *} p<0.0001$

561 
Figure 1

A Ubiquilin1wT

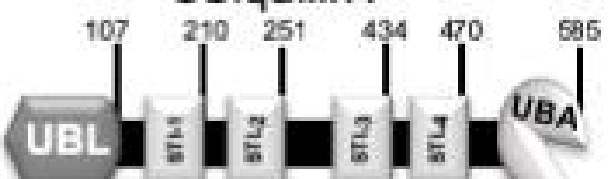

B

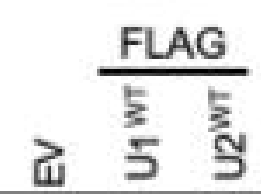

C

FLAG-

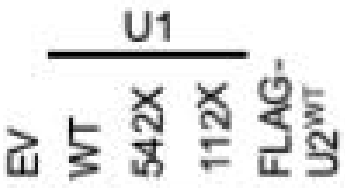

bioRxiv preprint doi: https://doi.org/10.1101/2020.06.03.131888; this version posted June 3, 2020. The cop/right holder for this prep int (which

was not certified by peer review) is the author/funder, who has grantedbibRxiv a license to displayl the preprint in perpetaity

Ubiquilin $1542 x$ available under aCC-BY-NC-ND 4.0-Internationallicense.

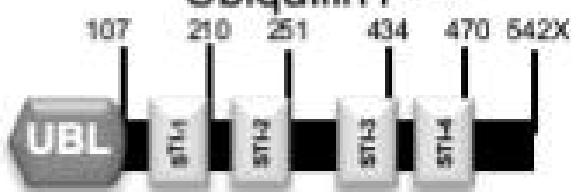

Ubiquilin $1^{112 x}$

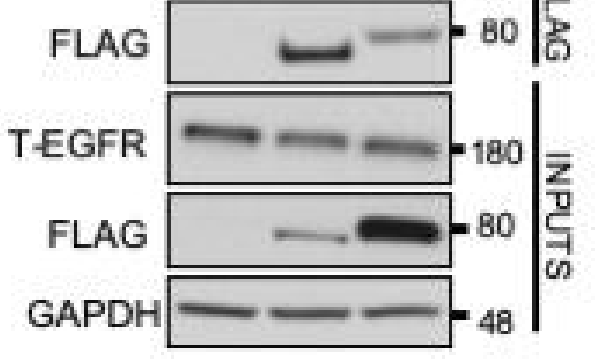

\section{Ubiquilin2WT}
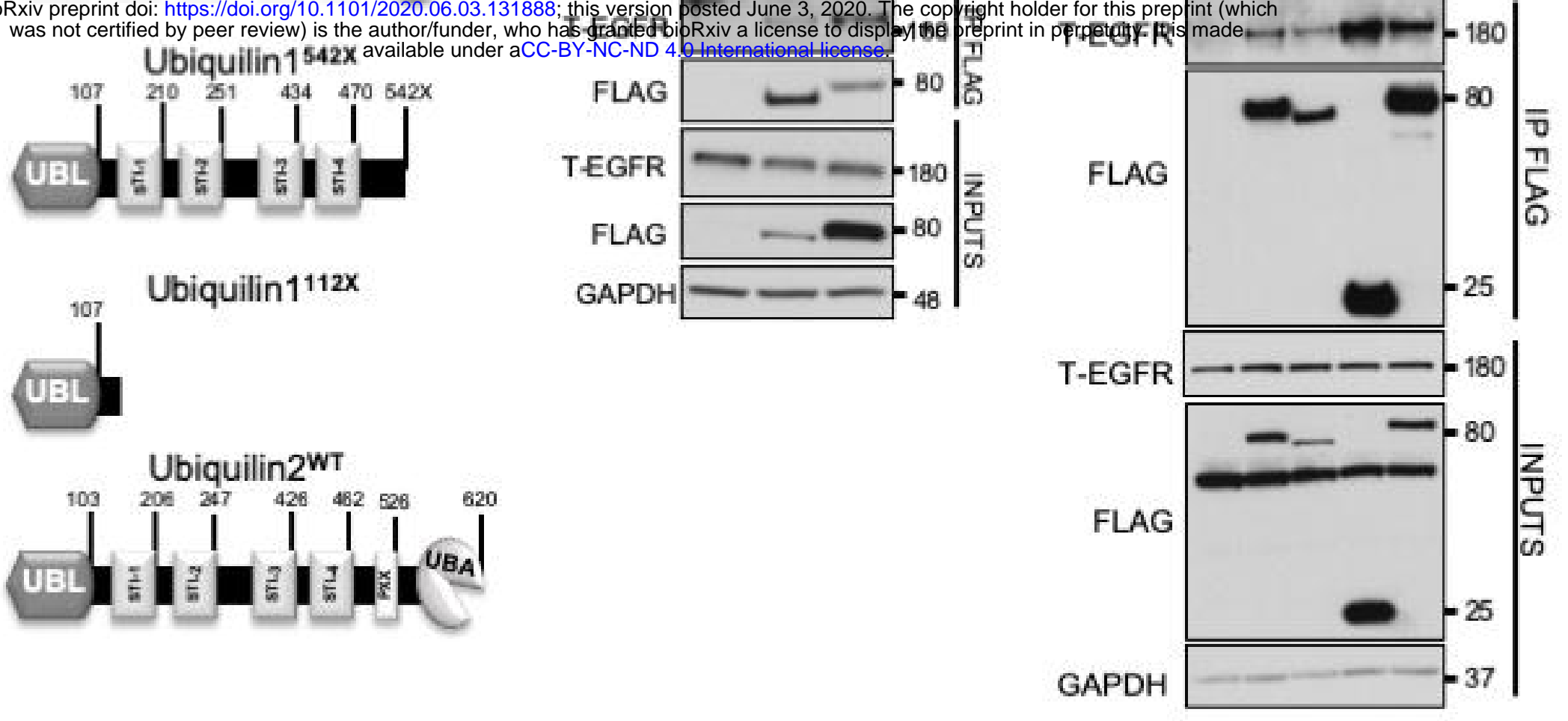
Figure 2

A

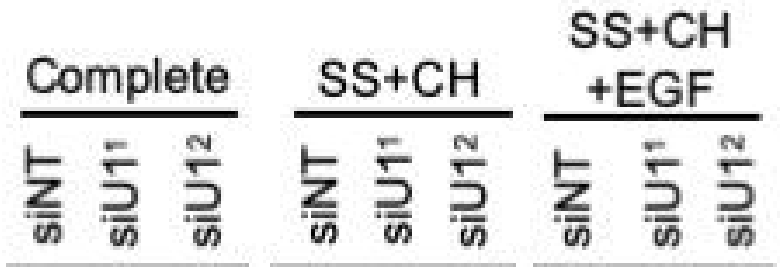

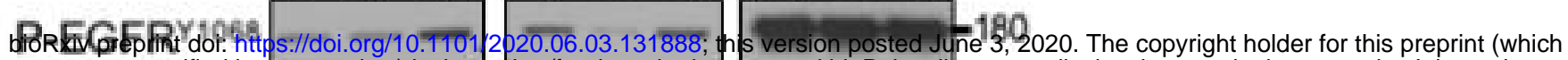
was not certified by beer review) is the author/funder, who has granted bioRxiv a license to display the preprint in perpetuity. It is made

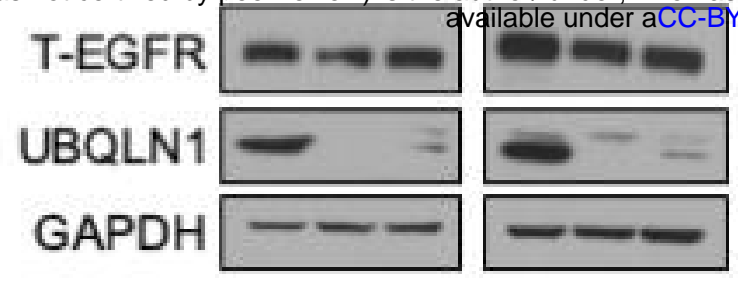

B
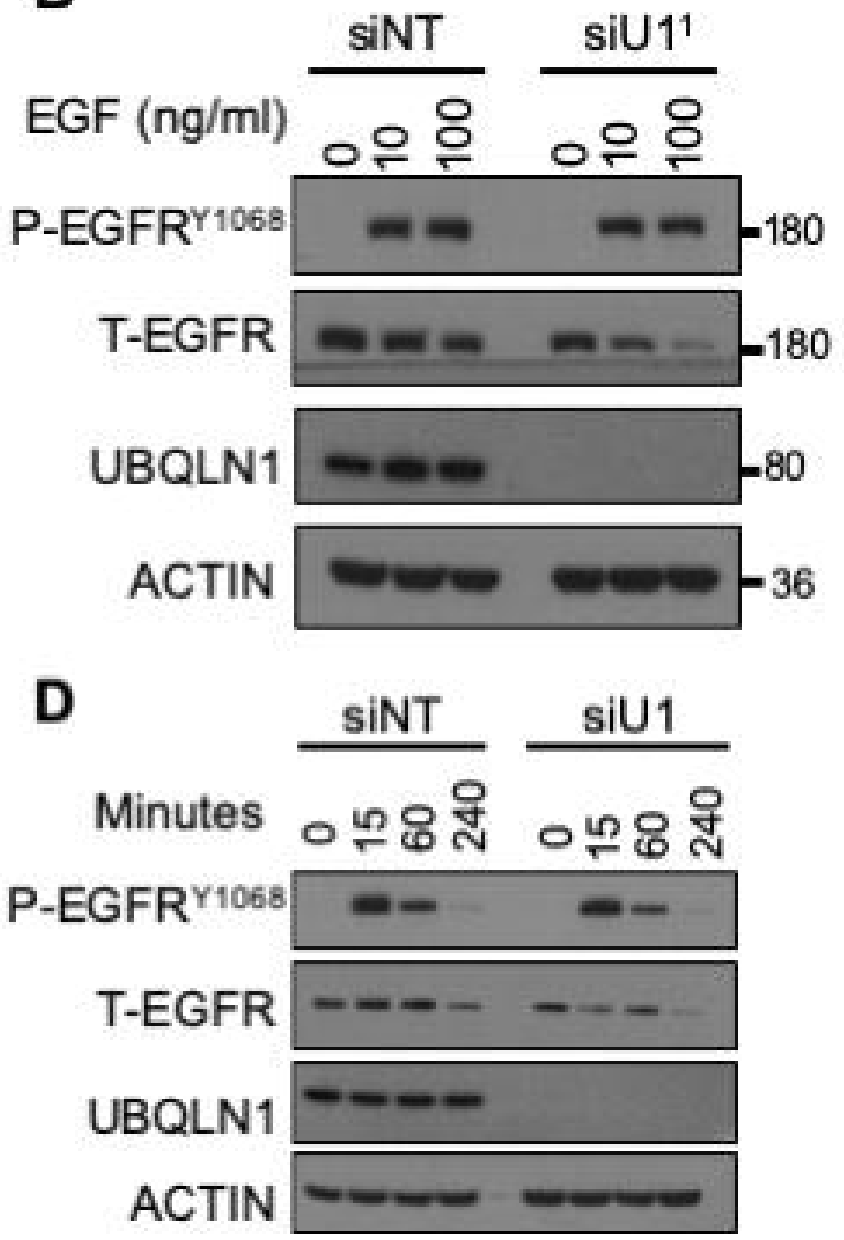

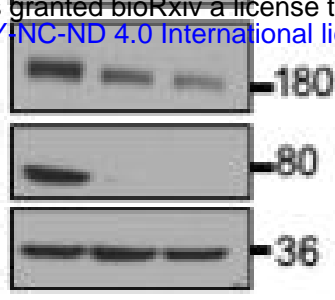

C

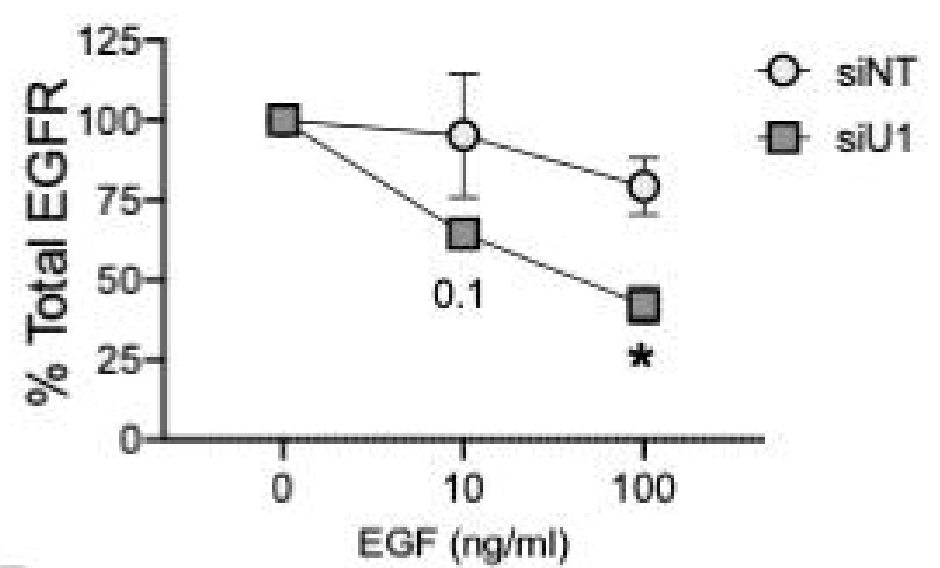

E

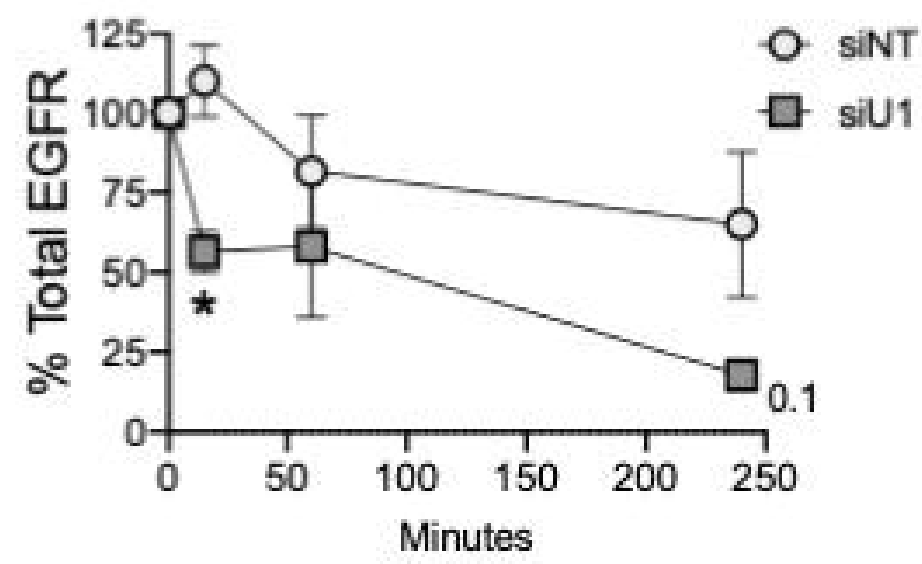


Figure 3

A

$$
\text { SS+ }
$$

B

$$
\text { SS EGF+ }
$$

Complete SS +EGF Erlotinib

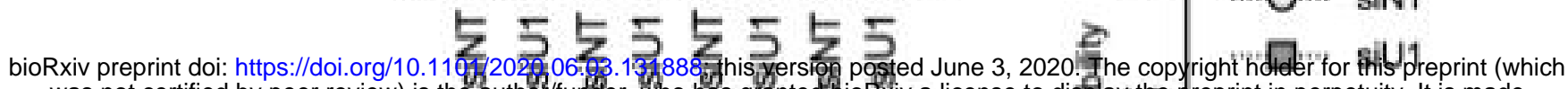

was not certified by peer review) is the author/funder, who has granted bioRxiv a license to display the preprint in perpetuity. It is made

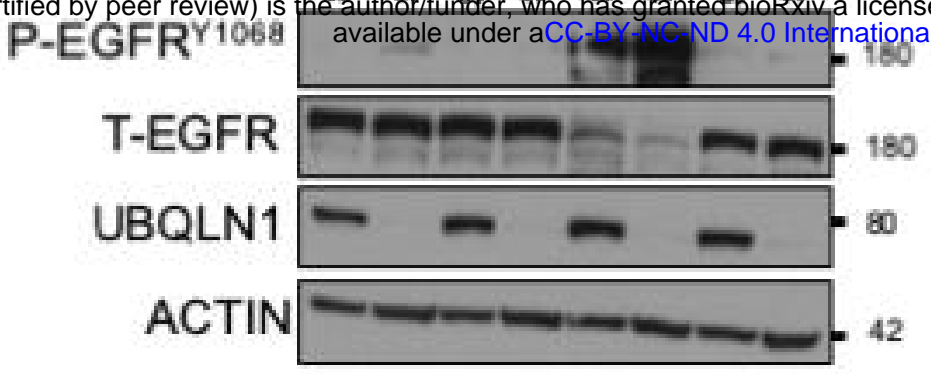

C

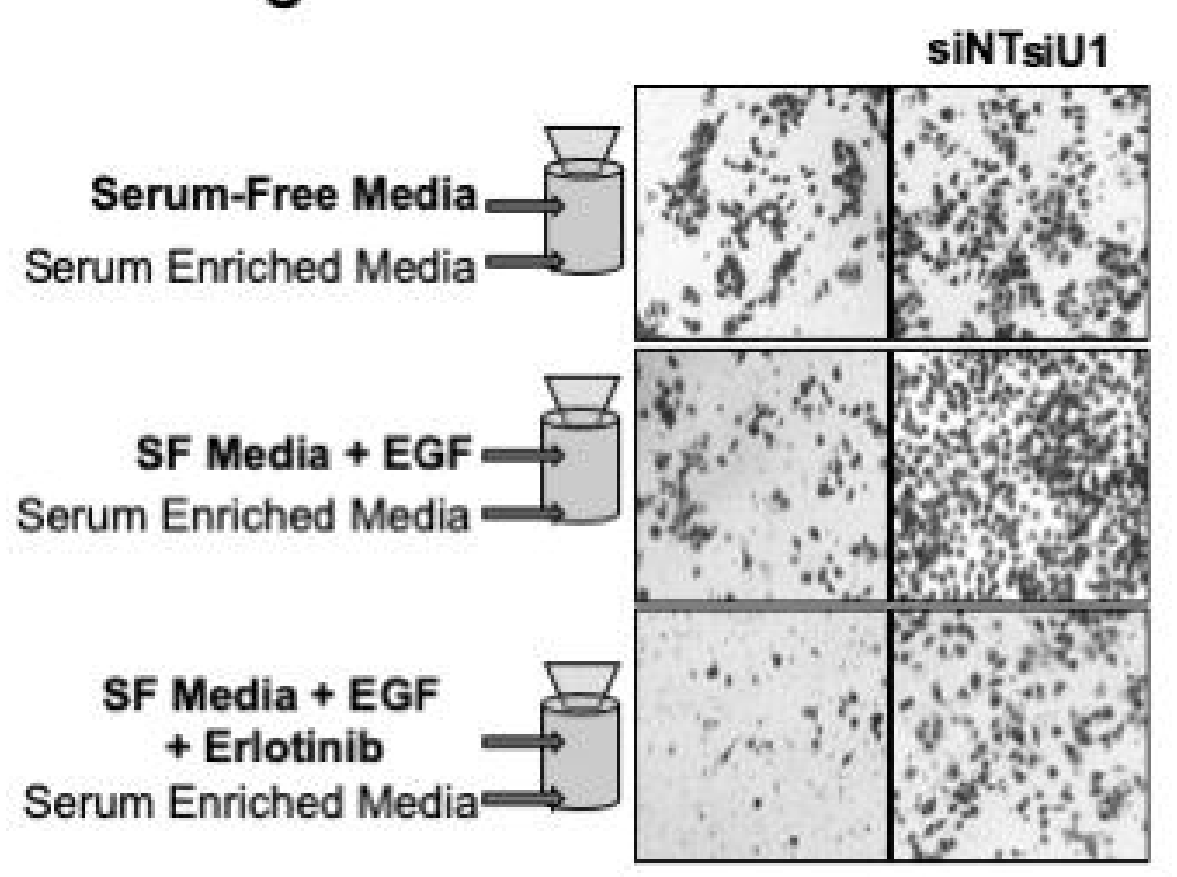

हु
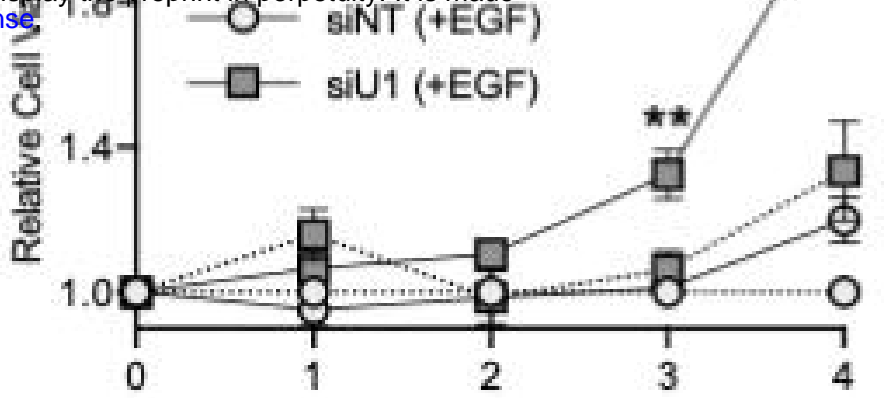

D

Days

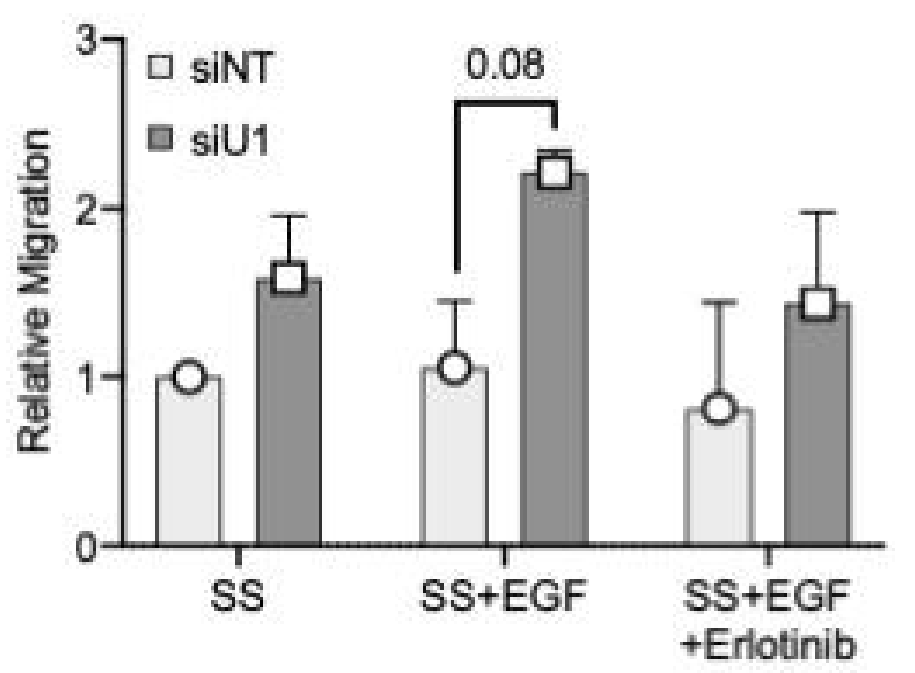


Figure 4

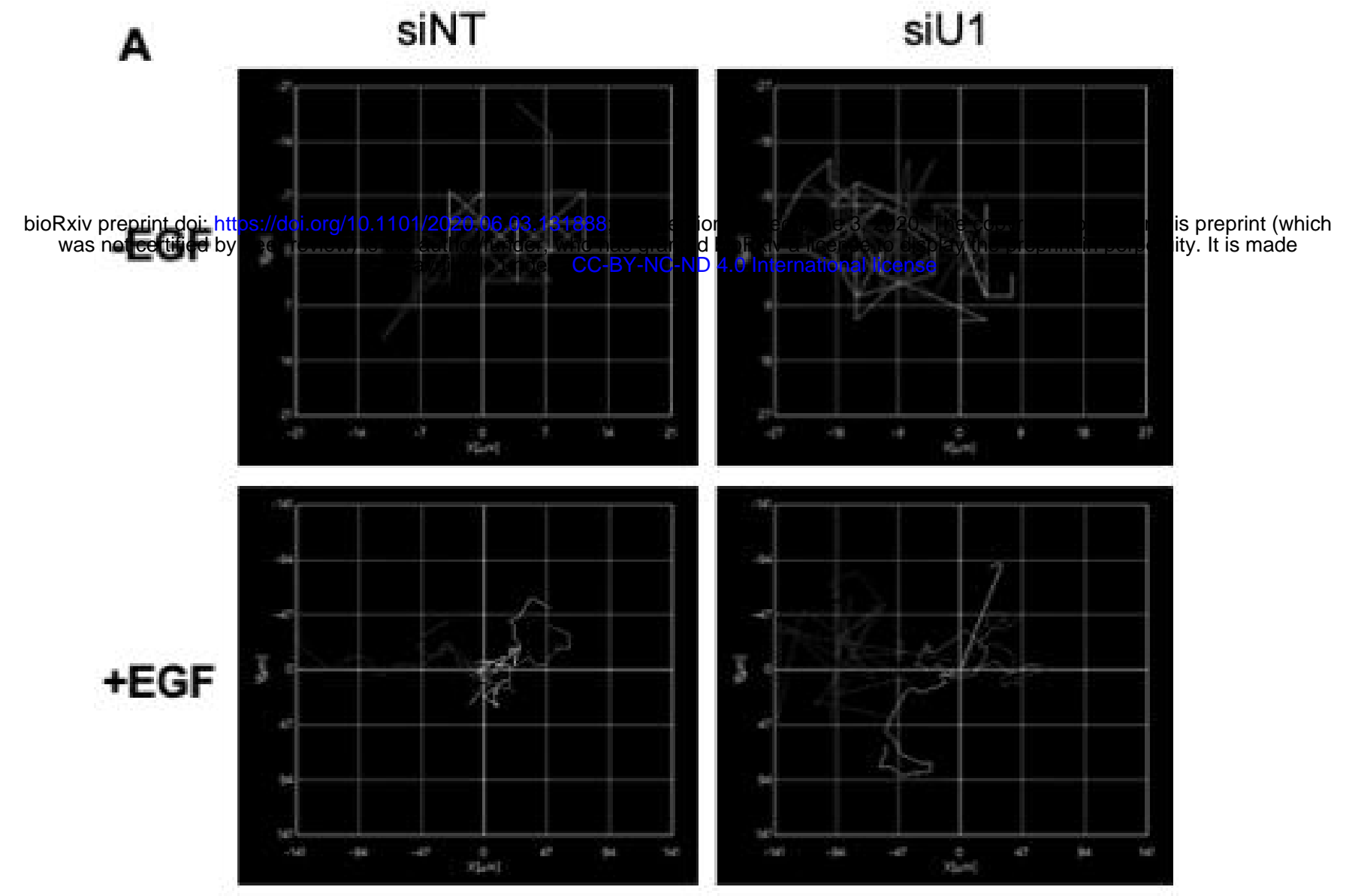

B

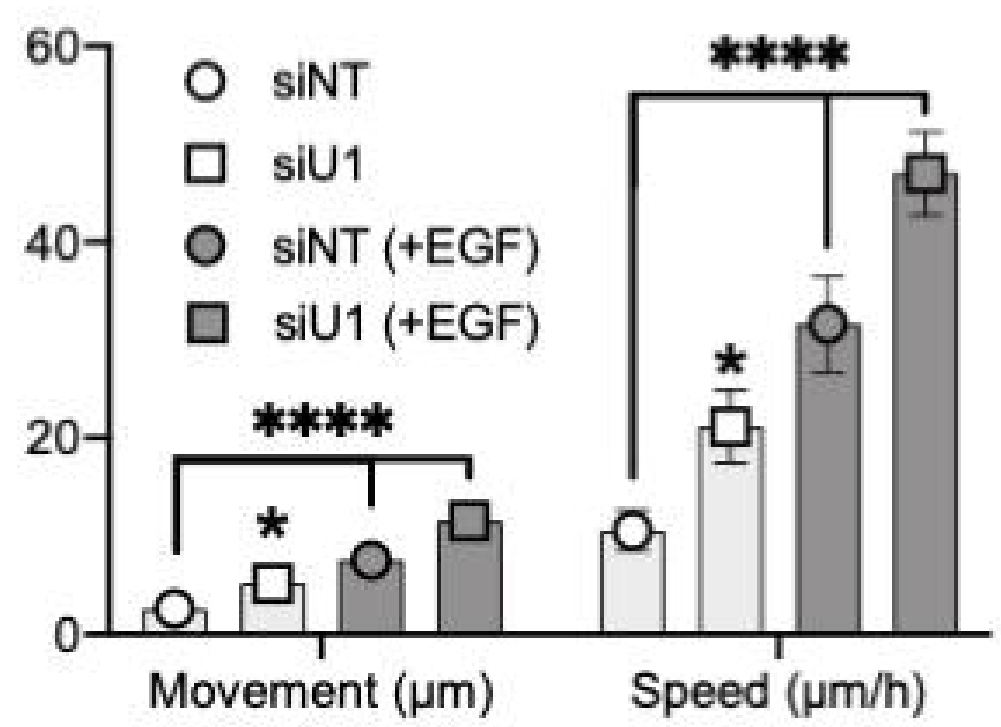

